\title{
A Case of Sequester and Involucrum Formation of the Fibula
}

\author{
Joanne C. Sierink ${ }^{\mathrm{a}}$, Niels W.L. Schep ${ }^{\mathrm{a}}$, Maaike P. Terra ${ }^{\mathrm{b}}$, Jan S.K. Luitse ${ }^{\mathrm{a}}$, J. Carel Goslings ${ }^{\mathrm{a}, \mathrm{c}}$
}

\begin{abstract}
A former healthy 22 year old man developed chronic hematogenous osteomyelitis of his right fibula. Ongoing disease unresponsive to antibiotic treatment led to operative debridement, despite the CT scan that showed no sequester formation. During the operation however, a large sequester of the fibula incorporated by an involucrum was found. We believe it is important to be cautious for this uncommon complication of osteomyelitis, even when radiologic imaging is suggesting otherwise. In case of progressive disease in a conservatively treated patient, surgical debridement is advised.
\end{abstract}

Keywords: Osteomyelitis; Hematogenous osteomyelitis; Sequester; Involucrum; CT

\section{Introduction}

The term osteomyelitis was first used by the French surgeon Chassaignac in 1852 [1]. It is defined as an inflammation of bone and bone marrow caused by pyogenic bacteria, mycobacteria or fungi [2]. If untreated, osteomyelitis may lead to formation of sequestra, i.e. pockets of dead cortical bone with abscess formation, and involucrum, new bone incorporating the sequestrum [3-6]. When the new bone is totally

Manuscript accepted for publication October 5, 2011

${ }^{\text {a}}$ Trauma Unit Department of Surgery, Academic Medical Center, Amsterdam, The Netherlands

${ }^{\mathrm{b}}$ Department of Radiology, Academic Medical Center, Amsterdam, The Netherlands

${ }^{\mathrm{c}}$ Correspondence authour: Goslings JC, Trauma Unit Department of Surgery, Academic Medical Center, Meibergdreef 9, 1105 AZ

Amsterdam, The Netherlands. Email:j.c.goslings@amc.nl surrounding the sequester, the involucrum is called 'Totenlade' (coffin) in German. This involucrum may or may not be adequate in supporting the limb structurally once the sequester is removed [7]. Sequestrum formation may result in large bone defects which may be overcome by involucrum formation, but these late results of chronic osteomyelitis are seldomly encountered in the industrialized world $[5,8,9]$.

We present a case of an adult and healthy young man from The Netherlands who developed chronic hematogenous osteomyelitis of his fibula. Because of ongoing disease unresponsive to antibiotic therapy, surgical debridement was performed and finally a sequester of the fibula, completely incorporated by an involucrum, was found. The patient was informed that data concerning his case would be submitted for publication and he consented.

\section{Case Report}

In June 2010, a 22 years old man with no relevant medical history, presented at the emergency department of a nonacademic, teaching hospital. He experienced pain in his right calf since one week. There was no history of preceding trauma or surgery. History taken from the patient revealed that he returned from a holiday to New-Zealand, the Fiji-islands, and Hong Kong a couple of weeks ago. There he suffered from small pustules on his left arm and right foot which recovered spontaneously. At physical examination done at first presentation, the patient had a temperature of $101^{\circ} \mathrm{F}(38.2$ ${ }^{\circ} \mathrm{C}$ ), a blood pressure of $144 / 87 \mathrm{mmHg}$ and a regular pulse of $96 \mathrm{bpm}$. The lungs were clear and heart sounds were normal. The abdomen was not tender and the liver and spleen were not enlarged. A warm, tensed and tender right calf was seen which was painful during active and passive extension of the foot. Extremity pulses were normal.

Laboratory testing was suggestive of infection with a leukocyte count of $20.2 \times 10^{9} / \mathrm{L}$ (normal $3.4-10.0 \times 10^{9} / \mathrm{L}$ ) and a C-reactive protein level of $100 \mathrm{mg} / \mathrm{L}$ (normal, $0-10$ $\mathrm{mg} / \mathrm{L}$ ). Ultrasonography was performed to exclude deep venous thrombosis. No thrombus was seen; however, diffuse swelling of the muscles was found. A supplementary radiograph of his right lower leg showed no abnormalities (Fig.

doi: $10.4021 / \mathrm{jmc} 367 \mathrm{w}$ 


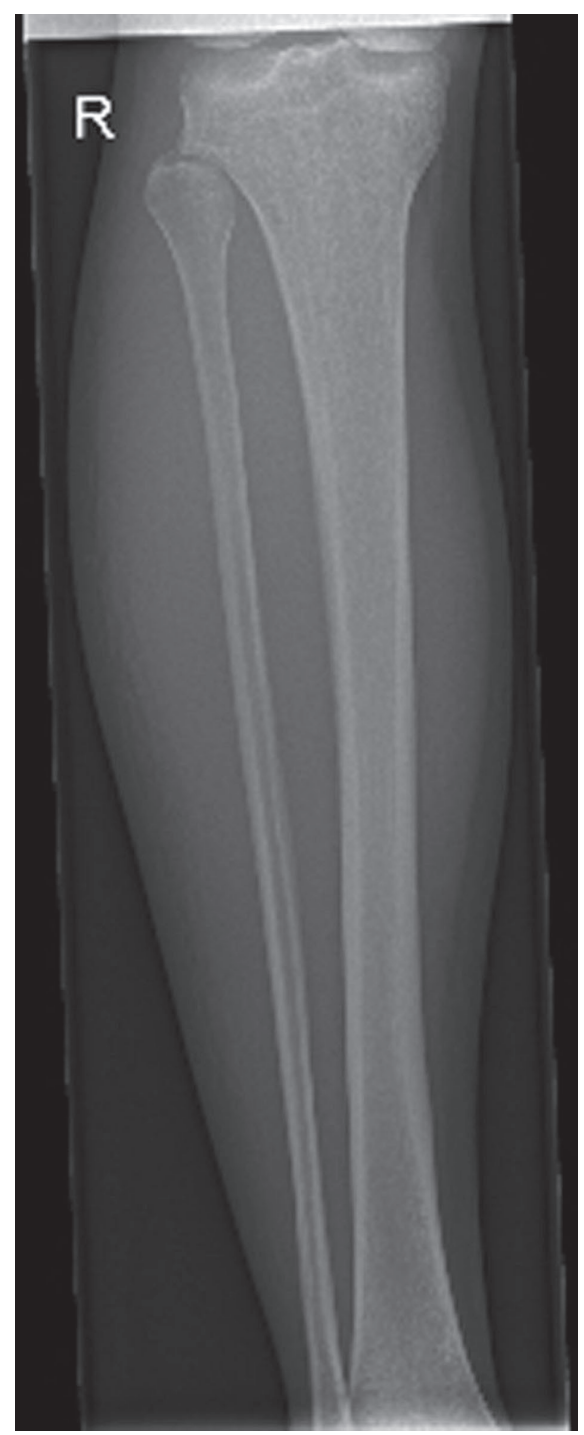

Figure 1. X-ray of the fibula of our patient at initial presentation without signs of osteomyelitis.

1). The swelling of the muscles was explained as reactive to severe physical activities he had undertaken the past few days and he was discharged with oral pain medication.

At day seven after the initial onset of symptoms, he presented to the same hospital with aggravation of his complaints. The existence of a compartment syndrome was clinically suspected and a four compartment fasciotomy of the right lower leg was performed. When releasing the fascia of the deep posterior compartment, a large amount of pus was discharged. Culture of the pus revealed a Staphylococcus Aureus. The patient was treated with Flucloxacilline for two weeks (one week intravenous, one week oral). The fasciotomy wound was closed with a split skin graft after three weeks and the patient was discharged.

Six weeks after the initial presentation, the patient was admitted again. He had a small pustule on his right calf and

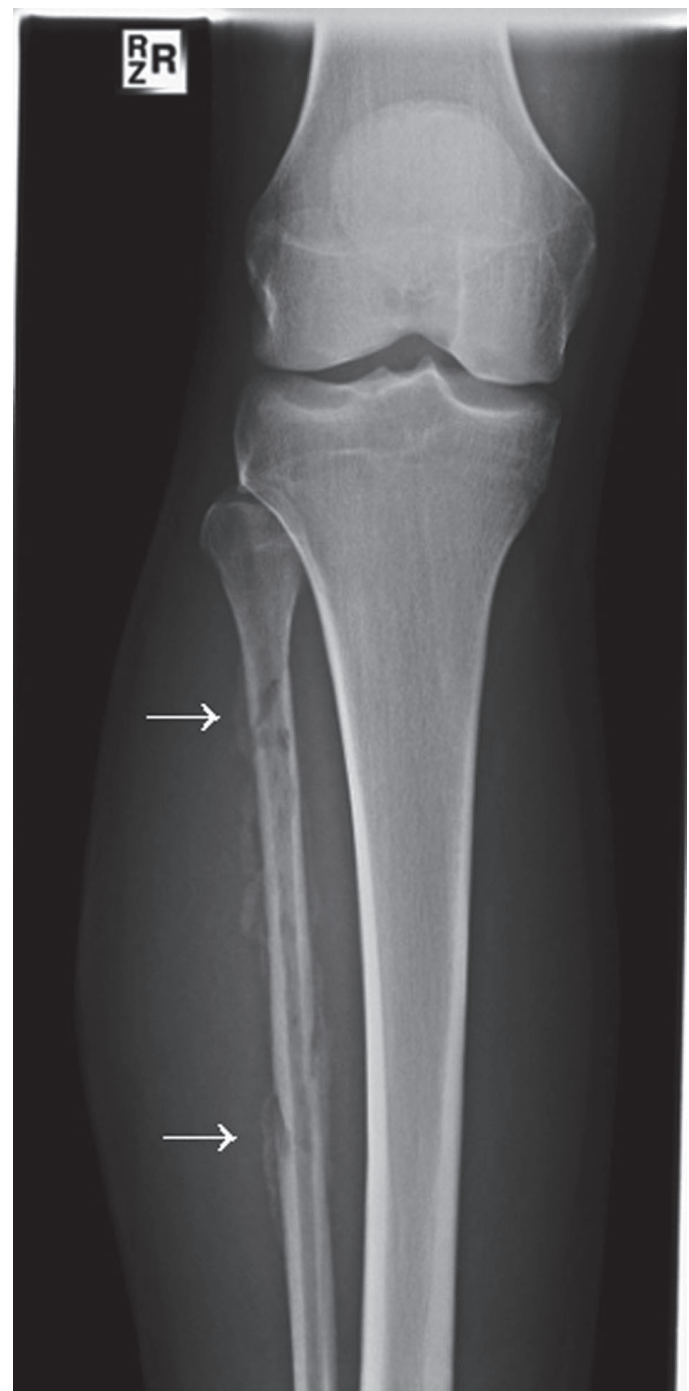

Figure 2. X-ray of the fibula 8 weeks after the onset of symptoms showing periostal reaction and partial lysis and interruption of the fibula.

redness of the skin surrounding the fasciotomy scar. No pain or fever was present. Both plain radiographic imaging (Fig.2) as well as magnetic resonance imaging (MRI) were suspicious of osteomyelitis of the fibula.

The patient was referred to our institution where a subsequent computed tomography (CT) confirmed the diagnosis osteomyelitis with destruction of the fibula and excessive periostal reaction with new bone formation (Fig. 3). Since there were no signs of a sequestrum, our treatment plan consisted of outpatient intravenous antibiotics (Flucloxacilline 1 gram six times daily during six weeks).

Six weeks after finishing the antibiotic therapy and four months after the initial onset of symptoms, the patient again presented with signs of infectious disease (infectious skin lesion at the right calf and a C-reactive protein level of 25 $\mathrm{mg} / \mathrm{L}$ ). A new MRI of the right fibula revealed severe mar- 


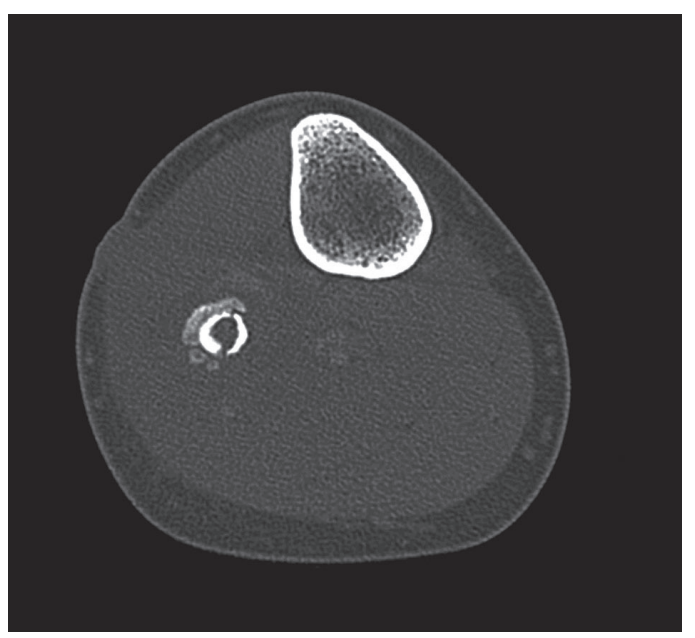

Figure 3. CT image of our patient which shows osteomyelitis and partial interruption of the cortex of the fibula and periostal reaction with new bone formation. No sequester was described.

row abnormalities with extreme periostal reaction in addition to extensive soft-tissue abnormalities with intramuscular abscess formation (Fig. 4). Based on the MRI findings and given the lack of clinical and laboratory evidence of improvement, surgical debridement of the fibula diaphysis was performed. A large abscess was found in the deep posterior compartment and material was collected for bacterial cultures. The lateral and anterior part of the fibula was found to be necrotic and a large sequester, incorporated by an involucrum was seen (Fig.5, 6). After debridement and sequestrectomy the patient was treated with bed rest with his right limb elevated. Gentamicine beads were left in the wound and since cultures of the bone revealed Staphylococcus Aureus sensitive for Flucloxacilline, he was once more treated with intravenous antibiotics for 6 weeks. The Gentamicine beads were replaced after 2 and 4 weeks and removed after

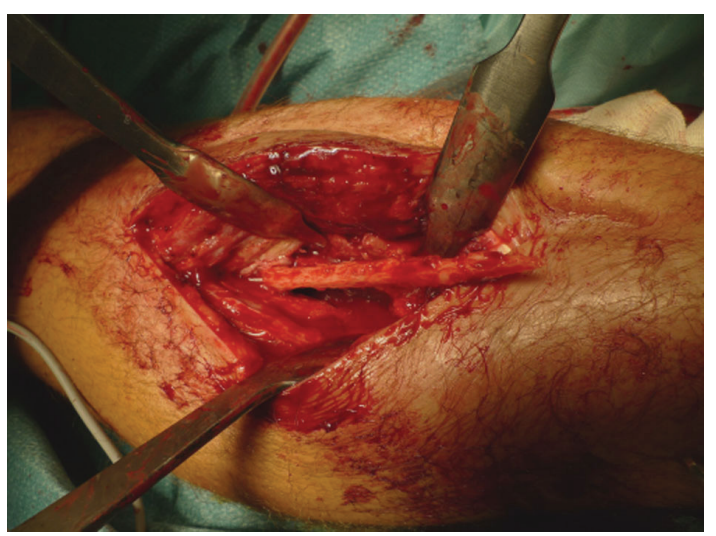

Figure 5. Sequestrum incorporated by an involucrum of the fibula.

6 weeks, following a negative culture. At the most recent follow-up visit, 5 months after hospital discharge, an excellent recovery was seen. X-rays showed bone formation at the site of the former sequestrum and the function of the leg was unrestricted.

\section{Discussion}

Waldvogel in 1970 was the first who described an osteomyelitis staging system [3]. He distinguished three etiologic routes, one of them being osteomyelitis secondary to hematological infection [3]. Distant foci of infection may lead to bacteraemia without clinical signs of sepsis. For patients with Staphylococcal osteomyelitis it is common to have a cutaneous infection such as pustules or furuncles in their medical history, as was the case in our patient [10]. The clinical pattern may evolve over months or even years and is characterized by a low-grade inflammation [9]. The development of necrotic bone and chronic osteomyelitis occurs roughly

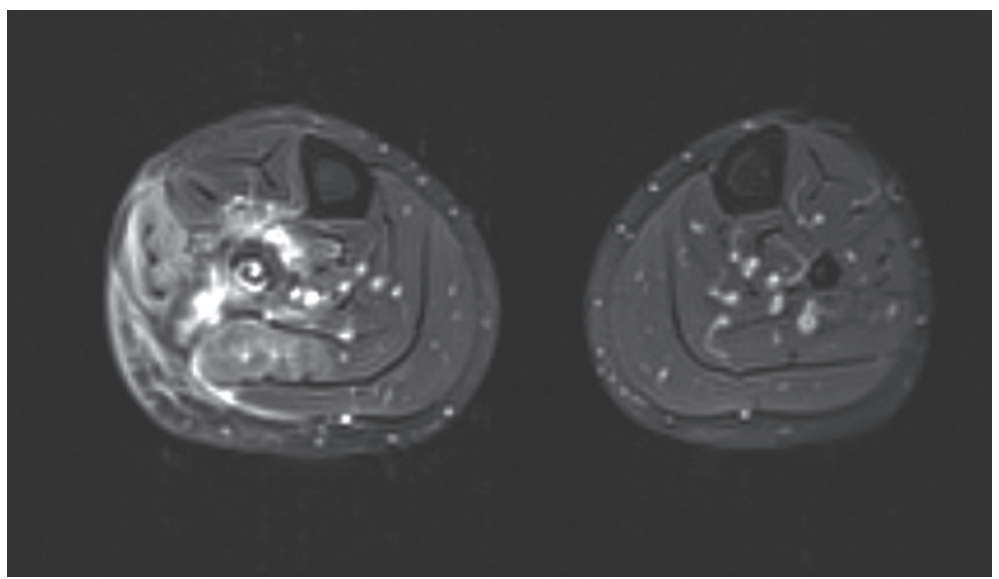

Figure 4. MRI image of our patient with extreme periostal reaction and extensive softtissue abnormalities with intramuscular abscess formation. 


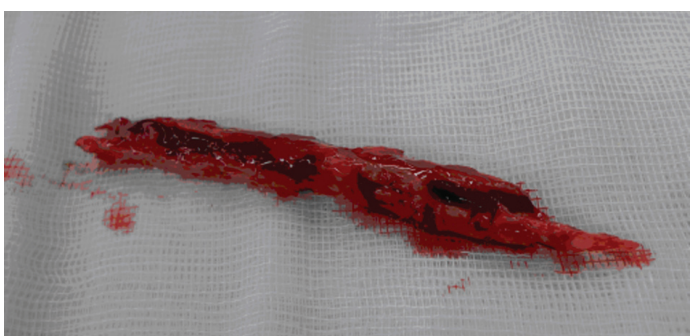

Figure 6. Removed sequestrum of the fibula.

after ten days [11] which explains the initial plain radiograph without abnormalities in the presented case.

Changes in plain radiographs can include soft-tissue swelling, osteopenia, scalloping of the cortex and periostal reaction $[6,9]$. CT scanning can be helpful in the identification of sequestra while MRI is more useful for soft-tissue assessment and revealing early bony oedema [9]. Both changes were seen in the presented case, however, only four months after the initial onset of symptoms.

Based on the CT-scan which did not show any sequestra, it was decided to treat this patient with intravenous antibiotics. This is in line with treatment guidelines based on the Cierny-Mader classification of osteomyelitis stage 1 (Table 1) $[11,12]$.

A useful algorithm of these treatment guidelines was presented in a 'current concepts review' by Lazzarini in 2004 [13]. However, a large sequester of the diaphysis of the fibula was found during the operation that was performed after four months delay. This discrepancy between the radiological imaging and what was found during the operation has, as far as the authors know, not been described before. The delay in diagnosis in the presented case was partly due to its indistinct presentation. Delay in diagnosis due to nonspecific symptoms was also described in hematogenous vertebral osteomyelitis and is reported to be two to four months [14], which is comparable with the delay described in our case. However, once referred to our institution the false-negative result of the CT directed us towards a conservative treatment while surgical debridement was in fact indicated.

Surgical management of osteomyelitis consists of two basic steps; debridement and obliteration of the subsequent dead space by soft tissue [15]. All sequestra must be removed and resection of scarred and infected bone and soft tissue is indicated [16]. Periostal stripping should be avoided because it may result in avascularity and the involucrum surrounding the infection can be left in place [6]. Debridement of the bone is performed until the 'paprika sign' is seen; a pin-point bleeding noted on the viable bone [12]. The dead space created following debridement may be replaced with viable vascularized tissue such as local muscle and skin flaps, or bone transport to fill large bone defects [9]. In the presented case, we decided to perform debridement with removal of the necrotic diaphysis of the fibula. Following debridement Gentamicine beads were changed until the the cultures showed no bacteria. No soft tissue was necessary to cover the dead space.

\section{Conclusion}

The lesson learned from this patient is that due to involucrum formation a CT scan can be false-negative regarding sequesters. We believe it is important to be cautious for this uncommon complication of osteomyelitis, even when radiologic imaging is suggesting otherwise. In case of progressive symptoms in a conservatively treated patient with osteomyelitis, surgical debridement is advised.

\section{Grant}

There were no sources of funding for this research.

Table 1. Cierny-Mader Classification System of Adult Osteomyelitis [12]

\begin{tabular}{|c|c|}
\hline Anatomic type & Physiologic host \\
\hline $\begin{array}{l}\text { 1: Medullary osteomyelitis. } \\
\text { Nidus is endosteal. }\end{array}$ & A: Normal host/immune system/vascularity. \\
\hline $\begin{array}{l}\text { 2: Superficial osteomyelitis. } \\
\text { Limited to the surface of the bone. }\end{array}$ & $\begin{array}{l}\text { B: Bs systemic compromise. } \\
\text { B1 local compromise. } \\
\text { Bs1 systemic and local compromise. }\end{array}$ \\
\hline $\begin{array}{l}\text { 3: Localized osteomyelitis. } \\
\text { Full thickness of the cortex is involved. }\end{array}$ & $\begin{array}{l}\text { C: Treatment morbidity worse than present } \\
\text { condition. }\end{array}$ \\
\hline
\end{tabular}




\section{Conflict of Interest}

The authors declare they have no conflict of interest.

\section{References}

1. Chassaignac E. De l'osteo-myelite. Bull Mem Soc Chir. 1852;431-436.

2. Conterno LO, da Silva Filho CR. Antibiotics for treating chronic osteomyelitis in adults. Cochrane Database Syst Rev. 2009(3):CD004439.

3. Waldvogel FA, Medoff G, Swartz MN. Osteomyelitis: a review of clinical features, therapeutic considerations and unusual aspects. 3. Osteomyelitis associated with vascular insufficiency. N Engl J Med. 1970;282(6):316322.

4. Waldvogel FA, Papageorgiou PS. Osteomyelitis: the past decade. N Engl J Med. 1980;303(7):360-370.

5. Daoud A, Saighi-Bouaouina A. Treatment of sequestra, pseudarthroses, and defects in the long bones of children who have chronic hematogenous osteomyelitis. J Bone Joint Surg Am. 1989;71(10):1448-1468.

6. Parsons B, Strauss E. Surgical management of chronic osteomyelitis. Am J Surg. 2004;188(1A Suppl):57-66.

7. Jones HW, Harrison JW, Bates J, Evans GA, Lubega N. Radiologic classification of chronic hematogenous osteomyelitis in children. J Pediatr Orthop. 2009;29(7):822827.
8. Blyth MJ, Kincaid R, Craigen MA, Bennet GC. The changing epidemiology of acute and subacute haematogenous osteomyelitis in children. J Bone Joint Surg Br. 2001;83(1):99-102.

9. Lew DP, Waldvogel FA. Osteomyelitis. Lancet. 2004;364(9431):369-379.

10. Wald ER. Risk factors for osteomyelitis. Am J Med. 1985;78(6B):206-212.

11. Mader JT, Norden C, Nelson JD, Calandra GB. Evaluation of new anti-infective drugs for the treatment of osteomyelitis in adults. Infectious Diseases Society of America and the Food and Drug Administration. Clin Infect Dis. 1992;15 Suppl 1:S155-161.

12. Cierny G, Mader JT, Pennick H. A clinical staging system of adult osteomyelitis. Contemp Orthop. 1985;10:17-37.

13. Lazzarini L, Mader JT, Calhoun JH. Osteomyelitis in long bones. J Bone Joint Surg Am. 2004;86-A(10):23052318.

14. Gasbarrini AL, Bertoldi E, Mazzetti M, Fini L, Terzi S, Gonella F, Mirabile L, et al. Clinical features, diagnostic and therapeutic approaches to haematogenous vertebral osteomyelitis. Eur Rev Med Pharmacol Sci. 2005;9(1):53-66.

15. Haidar R, Der Boghossian A, Atiyeh B. Duration of postsurgical antibiotics in chronic osteomyelitis: empiric or evidence-based? Int J Infect Dis. 2010;14(9):e752-758.

16. Eckardt JJ, Wirganowicz PZ, Mar T. An aggressive surgical approach to the management of chronic osteomyelitis. Clin Orthop Relat Res. 1994:298:229-239. 